\title{
Shaping the root canal system
}

\author{
M. Waplington*1 and A. S. McRobert ${ }^{1}$
}

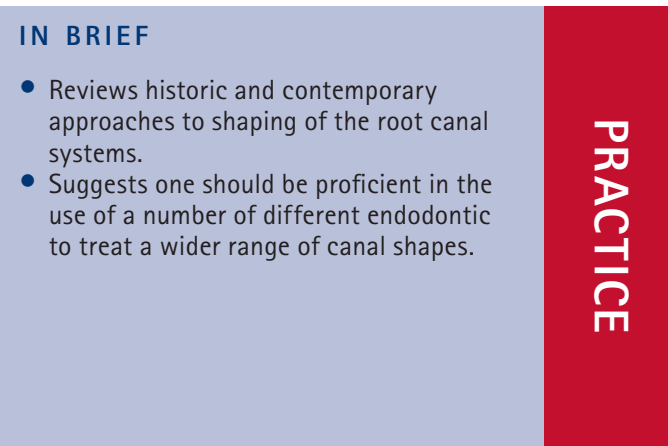

The speciality of endodontics encompasses numerous elements, but the use of a variety of instruments to shape root canal systems probably challenges the clinical time of practitioners more than any other. The aim of this article is to review some of the historic and contemporary approaches to this challenging practical objective.

\section{REASONS FOR THE MECHANICAL PREPARATION OF THE ROOT CANAL}

The use of mechanical instrumentation is accepted as being one of the most important steps in root canal treatment. ${ }^{1}$ It is also recognised as being one of the more difficult practical challenges in operative dentistry. The main objectives of canal preparation include:

- The removal of vital and necrotic pulpal tissue from the root canal space

- The removal of microorganisms and their products from the canal space

- The removal of infected dentine

- To create a space that can be both irrigated and medicated

- Allow predictable placement of a root canal filling material

- Maintain sound root and coronal tissue to allow effective restoration and function of the tooth.

Mechanical shaping must be accompanied by the thorough irrigation of the resultant space, a concept often referred to as 'cleaning and shaping', although many operators suggest that 'shaping and cleaning' is a more accurate term where files shape and irrigants clean. In practice these two objectives can be achieved simultaneously as part of an overall chemo-mechanical protocol.

Schilder ${ }^{2}$ is often cited as being one of the most influential authors in describing the form of root canal preparation shapes and

${ }^{1}$ Albert Road Dental Practice, Evesham, Worcestershire ${ }^{*}$ Correspondence to: Michael Waplington Email: mikewaplington@hotmail.com

\section{Refereed Paper}

Accepted 7 January 2014

DOI: $10.1038 /$ sj.bdj.2014.203

${ }^{\circledR}$ British Dental Journal 2014; 216: 293-297 is credited with describing important design concepts that still hold true today:

- A preparation that is continuously tapering with its largest diameter coronally and its narrowest diameter apically

- The root canal preparation should encompass the shape and original anatomy of the natural canal

- The original position of the apical foramen should be maintained

- The foraminal size of the preparation should be kept as small as practical (Fig. 1).

Unfortunately for clinicians the inherent complex anatomy of root canal systems ${ }^{3}$ including variations in length, curvature and diameter, as well as the presence of intricate canal anastomoses, provides a major challenge to the processes of canal shaping (Fig. 2).

\section{HISTORICAL APPROACHES TO CANAL PREPARATION}

Although the preparation of root canals has been described in literature since the start of the eighteenth century ${ }^{4}$ no systematic preparation sequence of instrumentation appears to have been proposed till $1961 .{ }^{5}$ For many years following this, canal preparation was completed using a sequence of stainless steel files or reamers in a serial step-back or incremental technique. ${ }^{6,7}$ This technique consisted of initially negotiating the canal to working length followed by enlargement of the apical portion of the canal, with shaping of the body of the canal being completed by using successively larger files at progressively shorter lengths (Fig. 3). This regularly proved to be a difficult procedure, which often utilised sharp ended instruments
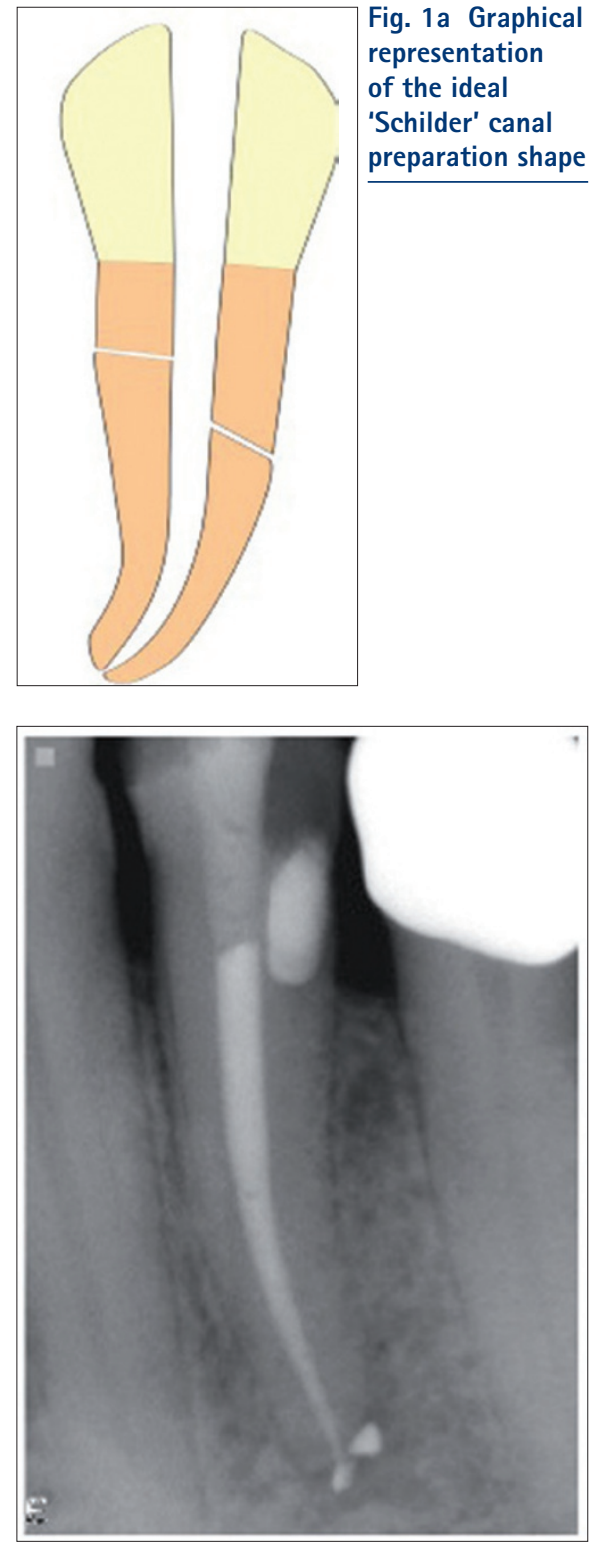

Fig. 1b Post-operative radiograph exhibiting a canal preparation with a continuously tapering shape and maintenance of original foraminal position 
in a push-pull filing motion. Disadvantages of this approach included considerable difficulty in achieving initial canal negotiation, premature blockage of canals with debris, inadequate irrigant solution penetration, and an increased incidence of canal ledging, transportation or perforation (Fig. 4). ${ }^{8}$ Often this technique proved to be time consuming and frustrating, especially where the original canal diameter was small, and the canal curvature more severe.

In response to the limitations of this technique an alternative step-down, or crown-down, approach to preparation was developed..$^{9,10}$ In essence this technique completely reversed the step-back approach, advocating the preparation of the coronal aspect of the canal first followed by sequentially smaller files penetrating the canal until working length is achieved. In addition controlled rotational movements of hand files, such as the 'balanced-force' technique $^{11}$ could be incorporated to minimise canal transportation. The crowndown approach offers several advantages over a step-back method:

- Enhances irrigation penetration and maintains a reservoir of irrigant coronally through which instruments can pass

- Minimises coronal flute binding of files, allowing enhanced control of the file tip and reducing procedural errors

- Reduces the straightening of pre-curved files once in the canal

- Enhances working length determination

- Reduces the possibility for extrusion of debris into the periradicular tissues

- Reduces the chances of inoculation of coronally placed pathogens into apical canal architecture

- Enhances straight line access for instruments into the canal system.

The crown-down technique is now widely accepted as the preferred approach to canal preparation, irrespective of the type of shaping instrument utilised. A summary of a typical instrument sequence is outlined in Table 1.

Classic approaches to canal filing have utilised ISO standardised 0.02 taper instruments by hand. These files have a diameter increase of $0.02 \mathrm{~mm}$ per millimeter from the file tip to the terminus of the $16 \mathrm{~mm}$ flute pattern (Fig. 5). The characteristic of these files to become inflexible in larger sizes and their tendency to produce relatively narrow preparations has been seen as a major disadvantage. ${ }^{12}$ These shortcomings have led clinicians and manufacturers to develop instruments that can complete canal shape more effectively and efficiently.

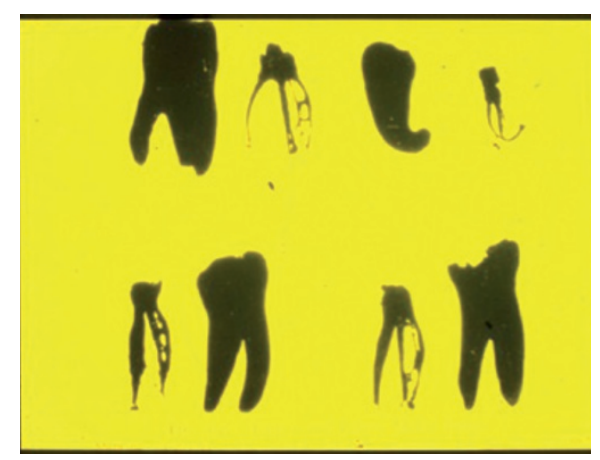

Fig. 2 The complex microscopic anatomy of the root canal system (after Hess ${ }^{3}$ )

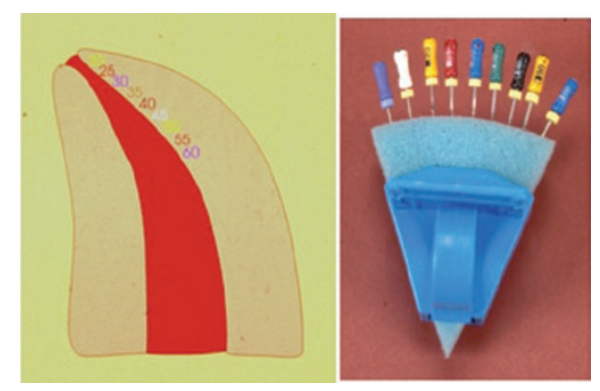

Fig. 3 Serial step-back procedure and file selection for preparation, and confirmation of deep canal shape

Table 1 Summary of a typical instrument sequence

Crown-down preparation sequence

Ensure straight line coronal access and confirm coronal canal patency: hand files sizes 8, 10, 15, 20

Flare coronal canal with Gates Glidden drills

sizes $4,3,2$ depending on canal diameter/curve. (Rotational speed 100-1500 rpm)

Careful exploration/scouting of canal to within two $\mathrm{mm}$ estimated working length

Working length determination (radiographic and electronic)

Complete deep canal shaping (hand files)

Finalise apical size/preparation

\section{NICKEL-TITANIUM (NITI) PREPARATION SYSTEMS}

Since the mid 1990s the introduction of root canal shaping instruments manufactured from nickel-titanium alloys have dramatically influenced preparation techniques. There are now dozens of different product types on the market, a range of choice that can lead to bewilderment for the dentist using such instruments for the first time. The metallurgic properties of NiTi alloys allows instruments to be manufactured with enhanced flexibility and shape memory compared to stainless steel products. This has obvious advantages when preparing canals with curvatures, as the potential for canal straightening is reduced. Another major advantage of NiTi instruments is the ability to make files with increased taper while retaining

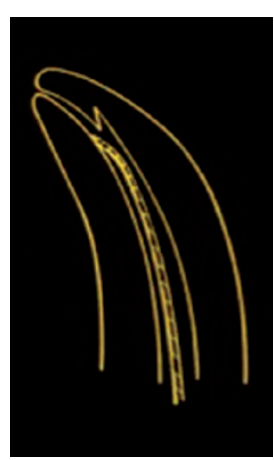

Fig. 4a Apical ledge formation is often created by the use of files with inappropriate tip size and lack of flexibility. Such blockages and ledges can be extremely difficult to negotiate

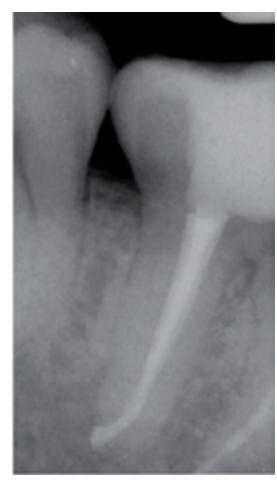

Fig. 4b Apical foramen transportation caused by over-instrumentation and length determination error

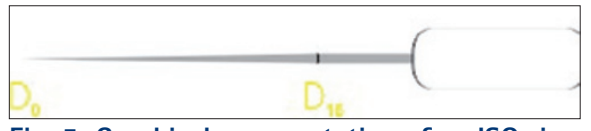

Fig. 5 Graphical representation of an ISO size 15 file. $D_{0}$ is $0.15 \mathrm{~mm}$ followed by $0.02 \mathrm{~mm}$ increases in diameter till $D_{16}$ is reached $(0.47 \mathrm{~mm})$

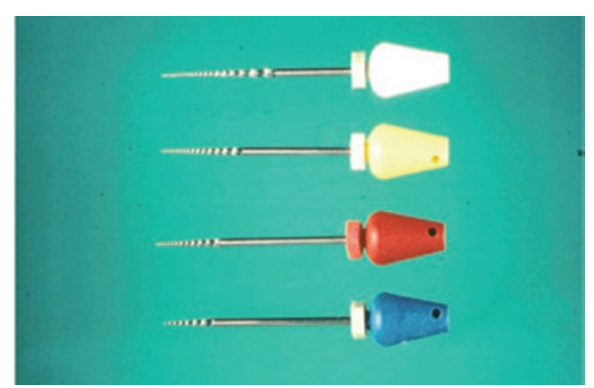

Fig. 6 Greater taper hand files. These files have increased taper rates: White -0.06 , yellow -0.08 , red -0.10 , blue -0.12 . Flexibility is maintained by $\mathrm{NiTi}$ alloy composition

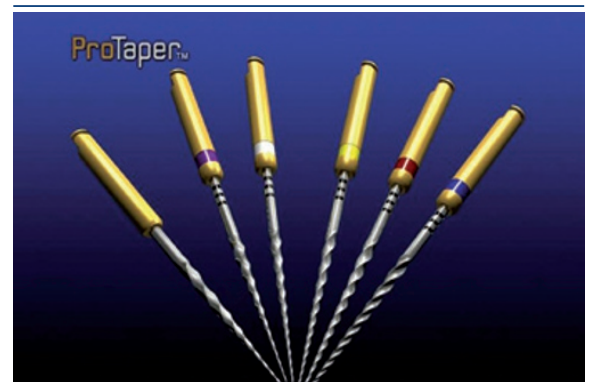

Fig. 7 The original ProTaper file series. These variable taper designs allowed a potentially reduced number of files for canal preparation

flexibility. Tapers ranging from a two-fold increase (0.04 taper) to a six-fold increase (0.12 taper) are now readily available (Fig. 6). Instruments like these have, until recently, 

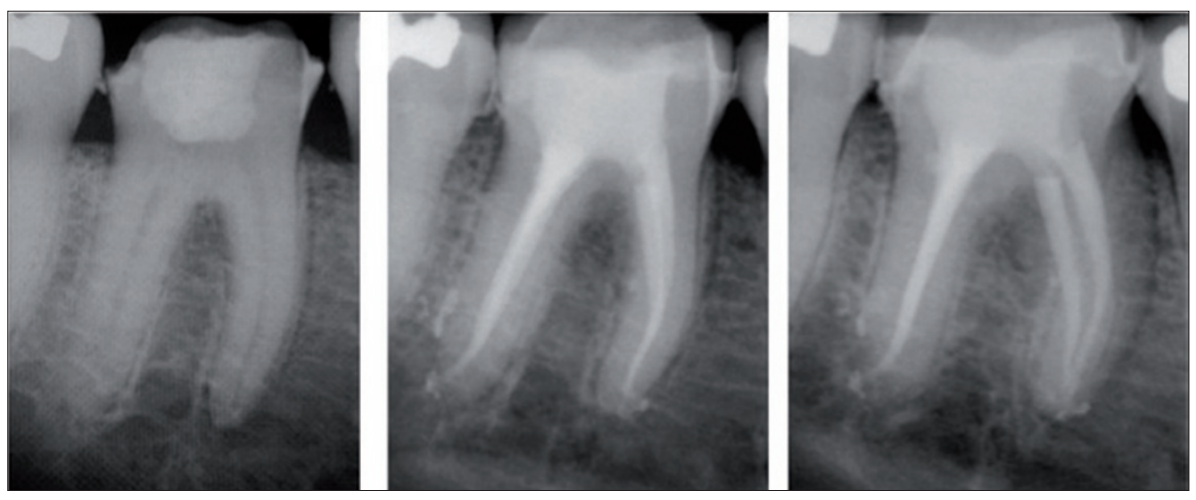

Fig. 8 Pre- and post-operative radiographs of a mandibular molar tooth prepared using the ProTaper system to finishing file F2. The mechanical objectives of canal shape have been observed
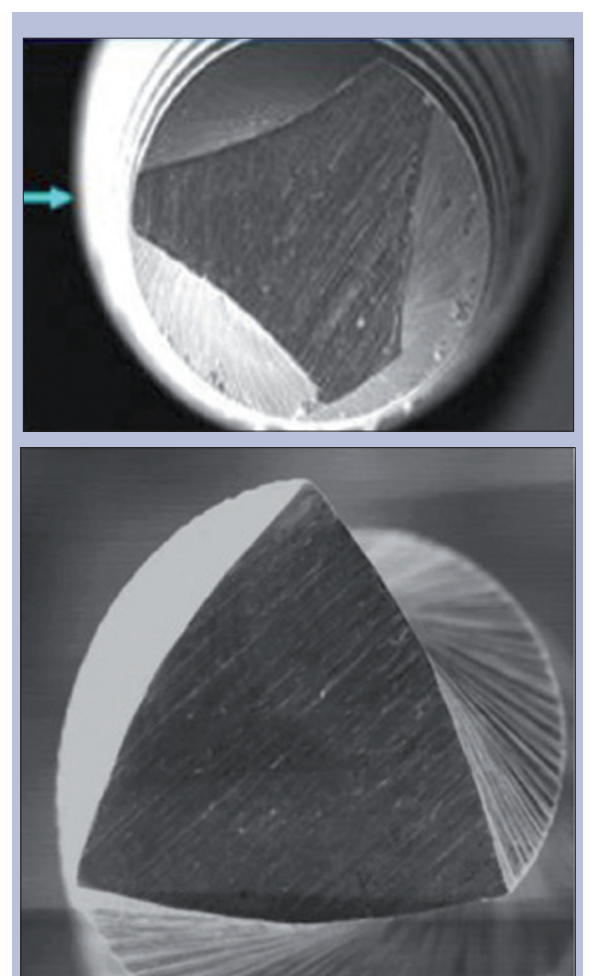

Fig. 9 Cross sectional SEM views of (a) a landed rotary NiTi file (Profile) and (b) a non-landed type (ProTaper)

been predominantly for use by hand, or driven through a 360 degree rotation by specialised speed/torque controlled electric motors. This provides the opportunity for canal preparation with regular and greater tapers, using fewer instruments often in less time (Fig. 7). ${ }^{13}$

NiTi rotary systems have developed a strong following in clinical practice and their use in general dental practice is becoming well established. ${ }^{14}$ While these instruments are no doubt extremely useful adjuncts to canal preparation, they alone cannot address every intricate variation in canal shape and the additional use of hand files must be considered essential to achieve thorough preparation. Following manufacturer's recommendations for use is a prerequisite for the avoidance of procedural errors such as file breakage. The following guidelines for rotary NiTi file usage apply to all manufacturers' systems:

- Proper straight line access should initially be achieved

- Rotary NiTi files are for canal enlargement of the canal, not negotiation

- NiTi files should follow a hand file created glide-path

- Use in a crown-down fashion

- The use of an appropriate speed/torque control motor is highly recommended

- Exert minimal apical pressure during canal instrumentation

- Lubricate/irrigate frequently

- Use each NiTi file within the canal for three to four seconds before withdrawing, cleaning and inspecting

- Regard NiTi files as single use items.

The last point in this list is particularly relevant to rotary NiTi files due to the increased levels of torsional and flexural stresses on the instrument. ${ }^{15}$ As a result of such stresses, rotary NiTi files should be discarded after each tooth preparation to ensure quality control of endodontic file efficiency. In the United Kingdom all endodontic files and reamers are now considered as single use. ${ }^{16}$

A substantial amount of research has been efficacy, preparation time, safety, and straightening effects of rotary NiTi systems. ${ }^{17}$ Overall conclusions from these studies state that:

- The use of rotary NiTi instruments in curved root canal systems results in a reduction of canal straightening and better centred preparations

- NiTi instruments alone are not capable of preparing all the canal walls

- As long as manufacturers' guidelines are followed the use of NiTi instruments can be considered safe. carried out on the shaping and cleaning
Practitioners should therefore be fully aware of the need for appropriate instrument training and familiarisation with different systems. The importance of thorough concurrent canal irrigation cannot be overstated. When the above factors are taken into consideration canal preparations can be achieved quickly and effectively, while maintaining the mechanical objectives of preparation shape previously noted (Fig. 8).

\section{ROTARY NITI \\ INSTRUMENT DESIGNS}

The detailed designs of the numerous rotary NiTi systems available are outside the scope of this article. However, there are basically two groups of design differences in regard to cross sectional shape: files with radial landed features and non-landed types. (Fig. 9). As a generalisation landed files allow a slower, but slightly more predictable preparation to their ability to stay centred in a canal. Nonlanded instruments have a more effective cutting action, and usually allow a more rapid preparation technique. Non-landed files require a higher level of operator care and experience in order to avoid preparation errors, especially in relation to working length determination.

\section{MEASURING WORKING LENGTH}

The question of where to finish the apical extent of a root canal preparation has divided the endodontic community for a number of years. Most endodontic practitioners will aim to prepare to the apical constriction of the canal, in essence where the pulpal tissues become peri-apical tissues. Locating this position, however, can be demanding as it cannot be identified radiographically, it is highly variable in relation to the radiographic apex of a root, and disease processes may alter or destroy it. ${ }^{18}$ In practice a combination of measurement techniques, including radiography, electronic apex location, tactile awareness, and the use of paper point measuring techniques often allows a more consistent method of measurement than a single technique alone. Modern electronic apex locators are now so consistently accurate that they form an invaluable aid to endodontic treatment.

\section{LIMITATIONS OF ROTARY NITI INSTRUMENTATION}

If correct procedure is followed, many root canals can be effectively shaped using rotary NiTi files. A 'golden rule' is to explore the canal system using small, flexible hand files, usually sizes 8,10 and 15, before inserting a rotary file to the same length. This 'scouting' of the canal can provide valuable tactile 

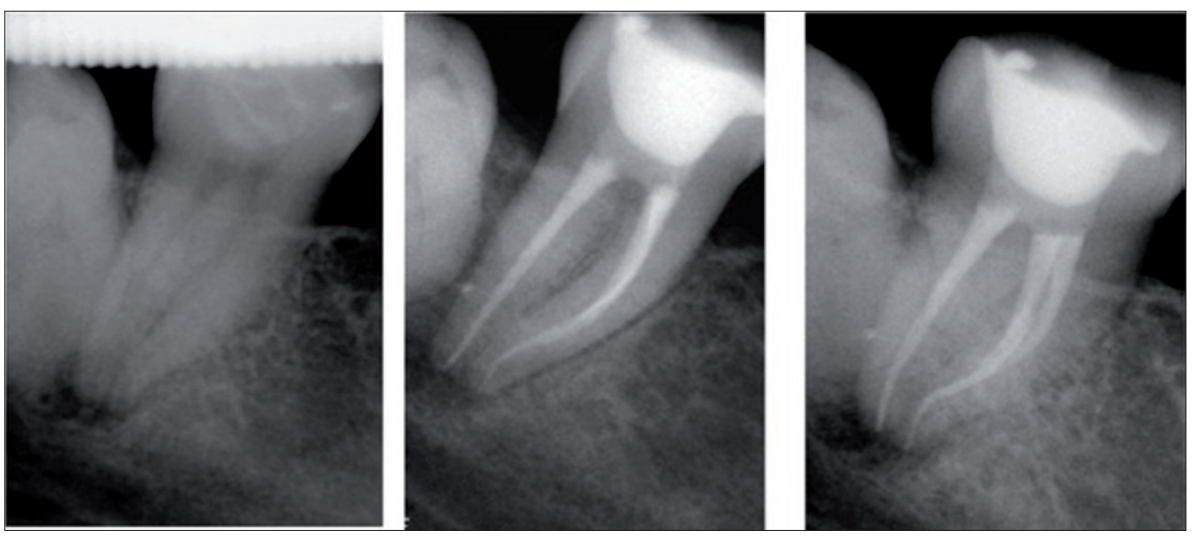

Fig. 10 Radiographic sequence showing a canal system with a distinct re-curvature. Preparation of such shapes can be a challenge for rotary NiTi files, and in this case the apical re-curve portion was prepared using hand instruments

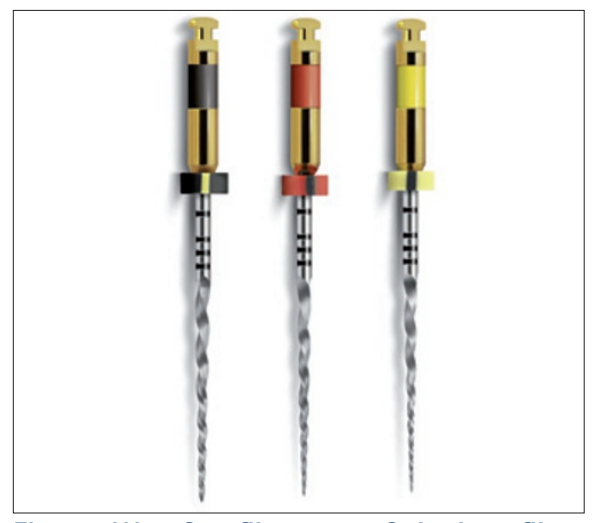

Fig. 11 WaveOne file system. Only three files are in this series: Small (yellow), primary (red) and large (blue)

information on the safety of the preparation case. Specifically, canals that exhibit sharp curvatures and re-curvatures, division, or convergence with abrupt change of direction, can provide challenging environments for rotating NiTi files due to increases in torsional and flexural stresses (Fig. 10). In these situations it is often prudent to complete preparation using pre-curved fine stainless steel hand files.

\section{THE 'SINGLE FILE' CONCEPT}

Many rotary NiTi systems require several files to achieve their preferred preparation shape. Along with concerns over file separation, decontamination and cost issues have driven manufacturers to try and reduce the number of NiTi instruments necessary to create an ideal shape. Some rotary NiTi systems are now available that have a greatly reduced number of files for example, Twisted Files $^{\circ}$ (Sybron Endo, Orange, CA), and others that utilise a single rotary instrument (One Shape;, Micro Mega ${ }^{\circ}$ Bescanson, France). Both these systems retain a full 360 degree rotational motion, and TF files utilise an altered state NiTi alloy that is purported to be more resistant to distortion and fracture. ${ }^{19}$ More recently the use of NiTi files in a reciprocating motion have been commercially developed that claim to allow canal shaping using only a single file (Wave One $^{\mathrm{TM}}$, Dentsply Maillefer, Ballaigues, Switzerland, and Reciproc ${ }^{\circ}, \mathrm{VDW}^{\circ}$, Munich, Germany). This concept was first reported by Yared $^{20}$ where a Protaper F2 finishing file was driven in a reciprocating fashion rather than through a conventional 360 degree rotation. The clockwise (144 degree) and anti-clockwise (72 degree) motions were controlled via an electronic motor, and the root canal was only pre-negotiated by an ISO 0.02 taper size 8 file. Such an approach was claimed to be effective at shaping and maintaining canal curvature. Additionally, advantages in reducing potential instrument
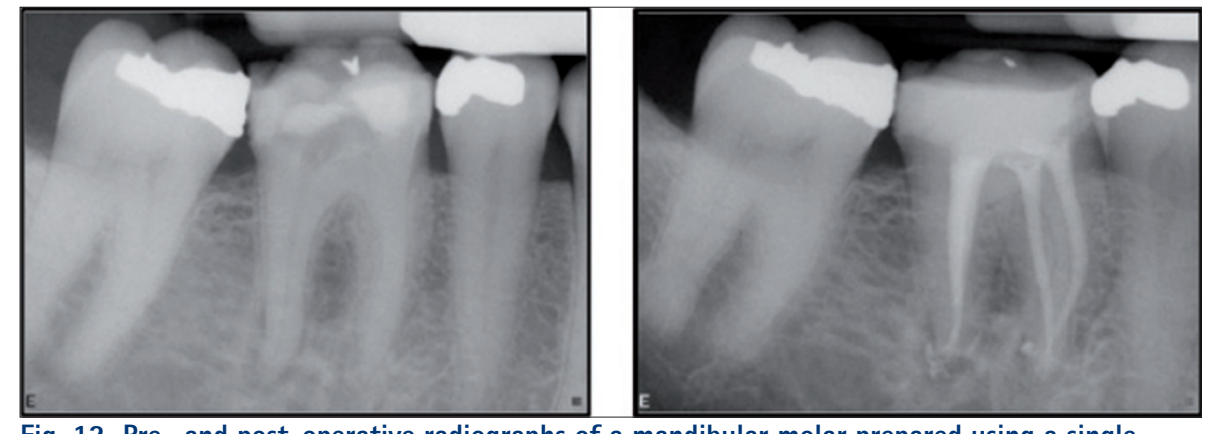

Fig. 12 Pre- and post-operative radiographs of a mandibular molar prepared using a single WaveOne primary file after 'glide-path' management

cross contamination and associated cost reductions were claimed.

These types of instruments have now been launched commercially, with a small number of preparation files to simplify the clinician's choice between small, medium and large canal sizes (Fig. 11). Both these reciprocating systems are made from a modified NiTi alloy (M-wire) and claim to be able to prepare adequate canal shape with a minimised risk of file fracture due to the reciprocating nature of the file movement. Reciproc ${ }^{\circ}$ is recommended for use without the need to create a 'glide-path' before its introduction into the canal, a claim that appears to have some experimental merit in roots with little or moderate canal curvature. ${ }^{21}$

Another study compared the shaping and cleaning potential of these new designs compared to 'conventional' rotary $\mathrm{NiTi}$ systems. $^{22}$ Both reciprocating systems maintained root canal curvature and were considered safe. The shaping time for the reciprocating files was naturally shorter due to the reduced number of files used, but it was noted that irrigation time of the canal should not be compromised by this reduced mechanical preparation time. Varying results regarding the amount and position of debris produced by reciprocating files has also been assessed $^{23}$ and further studies are merited to investigate the performance of this type of file.
There is no doubt that the introduction of reciprocating files has generated a high level of interest, especially in the general dental practitioner population. Their use in selected situations can produce consistently good results (Fig. 12), and the reduced number of files needed to prepare adequate shapes has obvious financial and time saving potential. The need to purchase a specialised motor capable of driving the reciprocating file correctly does initially offset some of these cost savings, but many of these dedicated units also allow the use of more conventional rotary systems, allowing the clinician to apply hybrid approaches to canal preparation using a combination of instruments. The authors of this article remain convinced that being proficient in the use of a number of different endodontic instruments allows a much more satisfying ability to address and treat a wider range of canal shapes.

\section{CONCLUSIONS}

The shaping of root canals is often considered by clinicians as a complex and challenging practical procedure. Historically there have been multiple approaches recommended to make it a more efficient and easier process, some of which have appeared to be contradictory to one another. The evolution of a crown-down approach and the introduction of advances in material 
design have revolutionised the clinical practice of many endodontists and general practitioners. The use of such mechanical method alone, however, has not impacted on the clinical success of endodontic procedures. It is essential that the mechanical preparation of the root canal system is backed up by a sound biologic knowledge of the endodontic disease process, and the application of complimentary antibacterial therapies to ultimately improve the clinician's ability to maintain the natural dentition. In this respect, the importance of thorough and effective canal irrigation cannot be over emphasised. Irrigation and its microbiological explanation are covered in another article within this endodontic issue.

1. Ruddle C. Cleaning and shaping the root canal system. In Cohen S, Burns R (eds) Pathways of the pulp. 8th ed. pp 231-292. St Louis: Mosby, 2002.

2. Schilder H. Cleaning and shaping the root canal. Dent Clin North Am 1974; 18: 269-296.

3. Hess W. Formation of root canals in human teeth. J Natl Dent Assoc 1921; 3: 707.

4. Lilley J D. Endodontic instrumentation before 1800 $J$ Br Endod Soc 1976; 9: 67-70.
5. Ingle J I. A standardized endodontic technique using newly designed instruments and filling materials. Oral Surg Oral Med Oral Pathol 1961; 14: 83-91.

6. Clem W. Endodontics in the adolescent patient. Dent Clin North Am 1969; 13: 483-486.

7. Weine $F$, Healeey L, Gerstein H, Evanson L. Precurved files and incremental instrumentation for root canal enlargement. J Can Dent Assoc 1970; 36: 155-157.

8. Weine $F$, Kelly $R$, Lio P. The effect of preparation procedures on original canal shape and on apical foramen shape. J Endod 1975; 1: 262-266.

9. Marshall FJ, Papin J. A crown down pressureless preparation root canal enlargement technique. Technique manual. Portland, Oregon: Oregon Health Sciences University, 1980.

10. Morgon L T, Montgomery S. An evaluation of the crown down pressureless technique. J Endod 1984; 10: 491-498.

11. Roane J B, Sabala C L, Duncanson M G Jr. The 'balanced force' concept for instrumentation of curved canals. J Endod 1985; 11: 203-211.

12. Saunders $E M$ M. Hand instrumentation in root canal preparation. Endodontic Topics 2005; 10: 163-167.

13. Saunders W P, Saunders E. Root canal instrumentation. In Bergenholtz G, Horsted Bindslev $P$, Reit C (eds) Textbook of endodontology. pp 236260. London: Blackwell Munksgaard, 2003.

14. Locke M, Thomas M B, Dummer P M. A survey of adoption of endodontic nickel-titanium rotary instrumentation part 1: General dental practitioners in Wales. Br Dent J 2013; 214: E6.

15. Yared G M, Bou Dagher FE, Machtou P. Cyclic fatigue of Profile rotary instruments after clinical use. Int Endod J 2000; 33: 204-207.

16. Government advises single use of endodontic instruments. Br Dent J 2007; 202: 442.

17. Hulsmann M, Peters $O A$, Dummer P M. Mechanical preparation of root canals: Shaping goals, techniques and means. Endodontic Topics 2005; 10: 30-76.

18. Ricucci D. Apical limit of root canal instrumentation and obturation. Part 1. Literature review. Int Endod 1998; 31: 384-393.

19. Gambarini G, Grande N M, Plotino G et al. Fatigue resistence of engine-driven rotary nickel-titanium instruments produced by new manufacturing methods. J Endod 2008; 34: 1003-1005.

20. Yared G. Canal preparation using only one NiTi rotary instrument: preliminary observations. Int Endod J 2008; 41: 339-344.

21. De Deus G, Arruda TE P, Souza E M et al. The ability of the Reciproc R25 instrumentto reach full root canal working length without a glide path. Int Endod J 2013; 46: 993-998.

22. Burklein S, Hinschitza K, Dammaschke T, Schafer E. Shaping ability and cleaning effectiveness of two single-file systems in severely curved root canals of extracted teeth: Reciproc and Wave-One versus Mtwo and Protaper. Int Endod J 2012; 45: 449-461.

23. Robinson J P, Lumley P J, Cooper P R, Grover $L M$, Walmsley A D. Reciprocating root canal technique induces greater debris accumulation than a continuous rotary technique as assessed by 3-dimensional micro-computed tomography. J Endod 2013; 39: 1067-1070. 